\title{
Validación psicométrica del inventario sistemas de creencias en Ecuador
}

\author{
Luis Alberto Bernal SARMIENTO CORREO
}

\author{
Corporación Neurotecnología para el Desarrollo Humano (NDH), Universidad de \\ Cuenca (Ecuador)
}

(Recibido el 25 de Noviembre de 2019, Aceptado el 31 de Marzo de 2020)

\begin{abstract}
RESUMEN: Los sistemas de creencias son conjuntos de mapas cognitivos que representan la realidad y se encargan de guiar a las personas en el funcionamiento social. Las primeras creencias construidas por el homo sapiens fueron las de tipo religioso, a través de estas explicaron sus orígenes. El objetivo de esta investigación fue analizar psicométricamente el Inventario Sistemas de Creencias Revisión 15 aplicado en una población de personas con enfermedades crónicas no trasmisibles en la ciudad de Cuenca-Ecuador. Este inventario mide creencias y prácticas religiosas y espirituales, y el apoyo social derivado de una comunidad que comparte esas creencias. El estudio contó con 153 participantes, el 61.4\% tenía cáncer, el $28.8 \%$ diabetes mellitus no insulinodependiente, el $7.2 \%$ otras enfermedades y el $2.61 \%$ hipertensión arterial primaria. El alfa de Cronbach fue de .897, la prueba Kaiser-Meyer-Olkin mostró un coeficiente de .900 y la prueba de Bartlett resultó significativa $p=<.000(X 2=1289.93 ; g l=105)$, la correlación entre elementos tuvo variaciones de .19 y .75. Se identificaron dos factores que explicaron el $58.61 \%$ de la varianza. Se encontró una relación positiva fuerte ( $r s=.569 ; p=.000$ ) entre la religiosidad del inventario y la frecuencia de uso de estrategias de afrontamiento religioso, uno de los dominios de la escala Estrategias de Afrontamiento Espiritual-Cuenca, cumpliéndose la validez concurrente. Se observaron diferencias significativas $(p<.05)$ entre los participantes, las mujeres puntuaron más alto en religiosidad que los hombres. Producto de este proceso de investigación se obtuvo el Inventario Sistemas de Creencias- Cuenca Revisión 15, versión que demostró validez y fiabilidad en una población de personas con enfermedades crónicas no transmisibles.
\end{abstract}

Palabras clave: psicología; ocupaciones relacionadas con la salud; factores religiosos; homínidos.

\section{Psychometric Validation of Belief Systems Inventory in Ecuador}

ABSTRACT: The belief systems are sets of cognitive maps that represent reality and are responsible for guiding people in social functioning. The first beliefs built by homo sapiens were those of a religious type, through which they explained their origins. The objective of this research was to psychometrically analyze the Inventory of Belief Systems Review 15 applied in a population of people with chronic noncommunicable diseases in the city of Cuenca-Ecuador. This inventory measures religious and spiritual beliefs and practices, and the social support derived from a community sharing those beliefs. The study 
included 153 people: $61.44 \%$ had cancer, $28.8 \%$ non-insulin-dependent diabetes mellitus, $7.2 \%$ other diseases and, 2.6\% primary arterial hypertension. Cronbach's alpha was .897, the Kaiser-Meyer-Olkin test showed a coefficient of .900 and the Bartlett test was significant $p=<.000(X 2=1289.93 ; d f=$ 105), the correlation between elements had variations of .19 and .75. Two factors were identified that explained $58.61 \%$ of the variance. A strong positive relationship ( $\mathrm{rs}=.569 ; p=.000$ ) was found between the religiosity of the inventory and the frequency of use of coping strategies, one of the domains of the Spiritual Coping Strategies-Basin scale, fulfilling the validity concurrent. Significant differences $(p<.05)$ were observed among the participants, women scored higher in religiosity than men. As a result of this research process, the Belief Systems Inventory - Cuenca Revision 15 was obtained, a version that demonstrated validity and reliability in a population of people with chronic noncommunicable diseases.

Keywords: Psychology. Allied Health Occupations. Religious Factors. Hominidae.

Correspondencia: E-mail: luis.bernals@ucuenca.edu.ec

\section{Introducción}

La finalidad de este estudio fue evaluar los sistemas de creencias religiosos-espirituales (SC) de personas con enfermedades crónicas no transmisibles (ECNT), para ello se examinó lingüística y psicométricamente la versión breve del Inventario Sistemas de Creencias Revisión 15, el nombre original del instrumento es Inventory Sistems of Belief (Holland et al., 1998). Para desarrollar este trabajo se plantearon dos hipótesis: (a) el inventario que evalúa los sistemas de creencias religiosas-espirituales diseñado en Estados Unidos se validará en una población de personas con ECNT que asisten a contextos de salud en Cuenca-Ecuador y (b) los elementos que integran los factores del Inventario Sistemas de Creencias versión Cuenca Revisión 15 (ISC-C 15 R) se agruparán en una estructura idéntica a la de la versión original.

\section{Sistemas de creencias}

El eje central del comportamiento religioso es la creencia en los espíritus que se supera con el desarrollo cultural de la ciencia. Las primeras creencias originadas por los humanos giran en torno a las proto-religiones, como el animismo, según el cual todos los objetos de la naturaleza poseen un espíritu (Morris, 1995).

En los periodos históricos que corresponden al homo habilis y erectus, la anatomía de los homínidos fue claramente primitiva junto con una cultura en la que los elementos simbólicos eran muy escasos (Rivera, 1998). Las creencias iniciales que construyó el homo sapiens se agruparon en sistemas de explicaciones de índole religiosa-espiritual. Esta forma de concebir el dinamismo del universo fue herencia de la experiencia de lo sagrado y del simbolismo (Ries, 2016), que al parecer estuvo presente en los ancestros humanos más remotos.

Un sistema es un conjunto de elementos dinámicamente estructurados, la totalidad del conjunto posibilita características que en parte son independientes de las propiedades que tienen cada uno de los componentes aislados (Feixas \& Miró, 1993). Además, es un cúmulo de ele-

mentos que tienen fuertes relaciones entre sí, estas interacciones mantienen al sistema, directa o indirectamente, unido de modo más o menos estable y cuyo comportamiento global busca algún tipo de objetivo (Arnold \& Osorio, 1998).

(C) Psy, Soc, \& Educ, 2020, Vol. 12(2) 
Los SC son interacciones sistémicas de agregados cognitivos que posibilitan construir historias cargadas de significado, por medio de ellas cada ser humano define de manera personal la realidad, entiende el mundo y su ambiente. Un SC no amerita tener un fundamento en la realidad, se adquiere por que brinda explicaciones adecuadas de modo consistente (Usó-Doménech \& Nescolarde-Selva, 2016).

Se elaboran a partir de la experiencia que requiere creencias previas y de la razón para ser asimilada. Los contextos son dinámicos debido a que cambian en función de las nuevas experiencias, así, se modifican las creencias y la manera de razonar. La realidad percibida se configura a través de sistemas de signos con la intención de dar sentido a la percepción, estos componentes son codificados gracias al aprendizaje de sistemas sociales, un significante puede ser icónico en un contexto de creencia, y ser simbólico, en otro distinto (Usó-Doménech \& Nescolarde-Selva, 2016).

\section{Procesamiento simbólico}

El humano es un homo religiosus desde los inicios de su devenir como especie (Eliade, 1999). Las primeras experiencias psicológicas de los homínidos fueron simbólicas, los símbolos son el resultado cognitivo de las transacciones iniciales de la morfofisiología de los hombres hábiles con la naturaleza (en sentido amplio).

Los esquemas cognitivos son estructuras nucleares de significado que maniobran la atención, la codificación y el recuerdo (Friedberg \& McClure, 2005). Estos esquemas contienen a las creencias más básicas y tempranas, resultado de la interacción entre el organismo del niño y su contexto. Otros componentes de la estructura neuropsicológica son lasoperaciones cognitivas y los productos cognitivos.

Los símbolos son estructuras cognitivas y procesos experienciales, a partir de ellos opera la fabricación simbólica para descubrir un sistema de significaciones personales y posibilitar la ocurrencia de los sentimientos en el terreno del mismo sistema. Ontológicamente, los seres humanos exploran y crean significados en el mundo (Saiz \& Amézaga, 2005).

La ciencia cognitiva de la religión posibilita patrocinar la convicción de que el funcionamiento cognitivo favorece condiciones en las que acepta creencias sobre seres sobrenaturales y promueve quehaceres motivados por la convicción en estos seres (Sztajer, 2013).

Los conceptos religiosos son combinaciones específicas de representaciones cognitivas: (1) de las representaciones religiosas que infringen ciertas expectativas de las categorías ontológicas y (2) de las representaciones religiosas que conservan ciertas características de las categorías ontológicas (Boyer, 2001).

Los símbolos desde frentes antropológicos son los componentes principales de la estructura del comportamiento religioso. Psicológicamente son imágenes, representaciones mentales primarias simples.

\section{Elaboración del instrumento psicométrico}

Desde los años 90s del siglo anterior, el interés por la investigación en religión y en espiritualidad tomó fuerza en territorios anglosajones, pues las creencias y prácticas de esta naturaleza favorecen en la calidad de vida de las personas que experimentan alguna enfermedad crónica (cáncer), así surgió la 
necesidad de elaborar instrumentos que evalúen estas esferas (Holland et al., 1998).

La primera versión del ISC desarrollado en Estados Unidos estuvo conformado por 54 elementos. Las poblaciones en las cuales se aplicó el inventario fueron hombres y mujeres, adultos con enfermedades y sanos, de tendencias ateístas, agnósticas y laicas y profesionales de la salud mental y de las órdenes religiosas (Holland et al., 1998).

La versión breve fue el resultado de la interacción entre el trabajo del personal del clero y el psiquiátrico, a partir de esta interacción se comprendió que los manejos psicoterapéuticos disminuyen la ansiedad y la depresión, también que los sistemas de creencias religiosos, respaldados por las visitas de una comunidad religiosa, ofrecen consuelo y permiten sobrellevar el diagnóstico de una enfermedad (Holland et al., 1998).

El ISC15-R surgió con intención de proporcionar una medida rápida de autoinforme en la investigación sobre la calidad de vida, los factores psicosociales y el afrontamiento ante la enfermedad, también para evaluar a población sin enfermedad. La esfera espiritual es un componente de la calidad de vida y la activación de este potencial, a su vez, mejora la percepción de la calidad de vida (Holland et al., 1998).

El marco conceptual para comprender la relación entre psicología y espiritualidad que condujo a los autores a elaborar el ISC 15-R fue la psicoterapia existencial del psiquiatra Irvin Yalom, quien teorizó sobre las cuatro preocupaciones supremas: muerte, libertad, soledad y sin sentido, como variables constantes de la experiencia humana. En el año de 1972, él organizó terapias de grupo integradas por personas que presentaban cáncer avanzado, dentro de estos procesos las aplicaciones clínicas giraban en torno a las preocupaciones supremas (Yalom, 1998).

Bajo los parámetros analizados, en el campo de la psicología de enlace y clínica ante los eventos que provocan un desbalance físico y psicológico como la enfermedad, el dolor o la muerte se guía al grupo para encontrar o retomar el sentido de vida, así por medio del sufrimiento, el humano tiene la capacidad de acceder a nuevos aprendizajes y también a la autorrealización.

Hasta hace unos años en Ecuador la psicología de la religión y la espiritualidad y la psicología de enlace no se habían relacionado a nivel de investigación. Estudios científicos efectuados en el austro ecuatoriano iniciaron el análisis de constructos numinosos moduladores de la personalidad en contextos de salud (Bernal, 2017, 2018).

\section{Estado del arte}

La versión ISC 15R está conformada por dos factores, que explicaron el $66.35 \%$ de la varianza. Se cumplió la validez convergente, divergente y pruebas de validez discriminante, también confiabilidad test-retest. Los alfas de Cronbach de los factores fueron: .92 factor I, .89 factor II y .93 para el instrumento total (Holland et al., 1998).

La versión mexicana evaluó a distintos grupos de personas como: pacientes, familiares y personal de salud. Se identificaron tres factores. Este instrumento presentó un alfa de Cronbach de .89 (Almanza, Monroy, Bimbela, Payne, \& Holland, 2000).

En el estudio efectuado en Israel con personas de comunidades religiosas, ultra-ortodoxas, nacionalistas y seculares, el análisis factorial se realizó por medio de validez convergente y discriminante. Se encontraron dos factores, cuya consistencia interna fue de .98 para el factor I y .96 para el factor II (Baider, Holland, Russak, \& De-nour, 2001). 
En la versión alemana ISC15-R D aplicada a personas del oeste y del este del país, la estructura de los dos factores del instrumento original no pudo ser replicada, tuvo que ser forzada, esta solución explicó el $80.9 \%$ de la varianza y la solución de un factor el 76.8\% de la varianza. Se encontró una consistencia interna total de .98 y para los dominios I y II esta fue de .97 y .94 respectivamente (Grulke et al., 2003).

La validación en Turquía con personas que tenían cáncer y recibían quimioterapia presentó dos factores que explicaron el $60.89 \%$ de la varianza. Los alfas de Cronbach reportados fueron: .98 para el dominio I, .93 para el dominio II y.98 para el inventario total (Erci \& Aktürk, 2017).

En una investigación llevada a cabo en Grecia con pacientes ambulatorios cristianos ortodoxos de ambos sexos, con un diagnóstico confirmado de tumores malignos. Los valores de la consistencia interna de la versión fueron .92 para la subescala de creencias y prácticas religiosas-espirituales y .85 para la subescala de apoyo social. Se encontró que las mujeres informaron niveles significativamente más altos de religiosidad en los dos factores (Assimakopoulos et al., 2009).

El instrumento validado en Italia se aplicó a usuarios (ambulatorios y hospitalizados) que experimentaban cáncer, la versión mostró una consistencia interna de .94 y .89 para los dominios I y II, correspondientemente (Ripamonti et al., 2010).

La versión colombiana investigó a personas distribuidas en dos grupos, estos son: con diagnósticos médicos (enfermedades crónicas) y estudiantes universitarios. Este inventario quedó conformado por dos factores que explicaron el 59.778 \% de la varianza, se eliminó el reactivo 6 que integra el inventario original. El alfa de Cronbach total fue de .92 (Riveros, Bernal, Bohórquez, Vinaccia, \& Quiceno, 2013).

Una nueva validación desarrollada en Alemania, en esta ocasión con un número representativo de familias, obtuvo una versión más breve, el ISC 6 elaborado mediante un análisis factorial exploratorio basado en el cuestionario ISC-15R-D (Grulke et al., 2003). Se encontraron dos dominios que mostraron una consistencia interna de .89 para creencias y prácticas, .92 para apoyo religioso y .94 para el inventarito total (Hampel, Brähler, Zenger, Albani, \& Heuft, 2019).

\section{Participantes}

\section{Método}

Fue un estudio cuantitativo de corte transversal y alcance descriptivo. Se basó en la validación lingüística y en la evaluación de las propiedades psicométricas de la versión ISC 15-R (Holland et al., 1998). La muestra estuvo constituida por 153 personas que presentaban enfermedades crónicas no transmisibles, con una edad media $=57.62$ y con una desviación típica $=14.3$. Se realizó en tres instituciones de salud de la ciudad de Cuenca: SOLCA, Hospital de Especialidades José Carrasco Arteaga y FASEC, durante el periodo de junio hasta diciembre de 2017.

Esta muestra se calculó de acuerdo a la proporción recomendada de un número específico de personas por cada elemento de la herramienta. Se ha recomendado una relación mínima de cinco personas por cada ítem de la escala y no menos de 100 individuos para efectuar un análisis factorial (Gorsuch, 1983; Hair, Anderson, Tatham, \& Black, 1998; Costello \& Osborne, 2005). 


\section{Instrumentos}

Se utilizó una ficha sociodemográfica para conocer: edad, enfermedad, diagnóstico, tiempo de enfermedad y religión. También el ISC compuesto por 15 ítems que se agrupan en dos factores: 1) sistemas de creencias y prácticas religiosas-espirituales y 2) apoyo social religioso-espiritual desarrollado por Holland en al año de 1996 en Estados Unidos. Es un instrumento tipo Likert con 4 opciones de respuesta que van de 0 a 3, a mayor puntuación mayor religiosidad tiene la persona.

La Escala Estrategias de Afrontamiento Espiritual Cuenca (EAE-C) tiene 17 ítems que forman cuatro dimensiones, las opciones de respuesta oscilan entre 0 (nuca utilizada) y 3 (a menudo utilizada). Para la validez concurrente se empleó el dominio de estrategias de afrontamiento religioso compuesto por cuatro ítems: 6,8,19,15 (Bernal, 2018).

\section{Procedimiento y análisis}

Los criterios de inclusión fueron: hombres y mujeres mayores de edad, con al menos un mes de diagnóstico y pacientes ambulatorios e internos con ECNT.

El análisis estadístico, se realizó con el programa estadístico SPSS 23, mediante análisis de varianzas y covarianzas. Inicialmente, se evaluó la consistencia interna del instrumento, mediante correlaciones y el análisis por ítem, además se realizó la prueba de fiabilidad mediante el alfa de Cronbach, posteriormente, se realizó un análisis factorial exploratorio mediante el análisis del componentes principales y el método de rotación ortogonal Varimax, se utilizó el índice Kaiser-Meyer-Olkin (KMO) y la prueba de esfericidad de Bartlett, adicionalmente, se realizó un análisis de validez concurrente mediante el coeficiente de correlación r de Pearson con la dimensión de -estrategias de afrontamiento religioso- que mide información similar y una validez de constructo por el método de grupos conocidos, para ello se usaron medidas de tendencia central y dispersión, se realizó la prueba no paramétrica de comparación de medias para dos grupos UMann Whitney y la prueba $\mathrm{H}$ Kruskal Wallis, la significación debía ser $\leq .05$ y la edición de tablas se realizó en Microsoft Excel 2016.

\section{Validación Lingüística ISC-C 15R}

El tratamiento lingüístico del ISC-C 15R inició con la traducción de la escala original del inglés al español, por un grupo de profesores quienes por medio de un consenso configuraron la primera versión en español del instrumento. Posteriormente, un equipo conformado igualmente, por profesores de la Universidad de Cuenca realizó la retro traducción de la escala. Desde el punto de vista de los núcleos gramaticales y semánticos otro grupo de profesionales estableció que la retro traducción coincidía con la versión original. Así se estableció la equivalencia gramatical y semántica de la escala siguiendo la estrategia de traducción inversa propuesta por Hambleton (1996).

Otros profesionales establecieron la equivalencia conceptual y de contenido, además, se trabajó con un grupo de estudiantes de la carrera de psicología clínica de octavo ciclo, quienes también concluyeron que el instrumento permite medir el constructo SC en la ciudad de Cuenca. La equivalencia técnica se estableció a través de reuniones con un $\mathrm{PhD}$ en Psicología y una persona versada en estadística. Una vez que finalizó el trabajo de la validación lingüística se efectuó el pilotaje del instrumento, posteriormente se recolectaron los datos para la validación 
psicométrica.

En la investigación de campo se procedió a informar a los participantes sobre los fines del estudio. En cumplimiento de las normas éticas, los participantes dieron su consentimiento informado para participar.

\section{Resultados}

El estudio se realizó con 153 personas diagnosticadas con enfermedades crónicas no transmisibles, el $61.4 \%$ fueron usuarios con cáncer, el $28.8 \%$ con diabetes mellitus no insulinodependiente, el $2.6 \%$ con hipertensión primaria y el $7.2 \%$ con otras enfermedades, con edades de entre 20 y 85 años $\left(M_{\text {edad }}=57.6, D E=14.3\right)$ y con un tiempo de diagnóstico de al menos un mes. La mayoría de participantes eran mujeres (mujeres $=60.8$ y hombres $=39.2 \%$ ), se encontraban casadas, pertenecían a la religión católica y tenía un nivel de instrucción académica secundaria.

Tabla 1. Estadísticos descriptivos

\begin{tabular}{|c|c|c|c|}
\hline & & \\
\hline & & $\mathbf{N}$ & $\%$ \\
\hline \multirow[t]{2}{*}{ Sexo } & Hombre & 60 & 39.2 \\
\hline & Mujer & 93 & 60.8 \\
\hline \multirow{4}{*}{$\begin{array}{l}\text { Nivel de } \\
\text { instrucción }\end{array}$} & Primaria & 51 & 33.3 \\
\hline & Secundaria & 61 & 39.9 \\
\hline & Superior & 35 & 22.9 \\
\hline & Sin escolaridad & 5 & 3.3 \\
\hline \multirow[t]{5}{*}{ Estado civil } & Soltero/a & 37 & 24.2 \\
\hline & Divorciado/a & 19 & 12.4 \\
\hline & Casado/a & 75 & 49.0 \\
\hline & Unión Libre & 7 & 4.6 \\
\hline & Viudo/a & 15 & 9.8 \\
\hline \multirow[t]{2}{*}{ Residencia } & Sector urbano & 108 & 70.6 \\
\hline & Sector rural & 44 & 28.8 \\
\hline \multirow[t]{5}{*}{ Religión } & Ateo & 1 & .7 \\
\hline & Católica & 131 & 84.5 \\
\hline & Cristiana & 10 & 6.6 \\
\hline & Cristiana evangélica & 7 & 4.7 \\
\hline & Testigo de Jehová & 4 & 2.6 \\
\hline \multirow[t]{4}{*}{ Diagnóstico } & Cáncer & 94 & 61.4 \\
\hline & Diabetes mellitus no insulinodependiente & 44 & 28.7 \\
\hline & Hipertensión primaria & 4 & 2.6 \\
\hline & Otro & 11 & 7.2 \\
\hline
\end{tabular}




\section{Fiabilidad interna y análisis factorial exploratorio}

La fiabilidad de consistencia interna del instrumento resultó aceptable con un alfa de Cronbach de .897 la prueba Kaiser-Meyer-Olkin (KMO) mostró un coeficiente de .900 y la prueba de Bartlett resultó significativa $p=.000\left(\chi^{2}=1289.93 ; g l=105\right)$, la correlación entre elementos tuvo variaciones entre .19 y .75, mostrando una correlación media entre elementos de .43. En la tabla 2 se muestra la correlación entre elementos.

Tabla 2. Correlación entre elementos del Inventario Sistema de Creencias en Cuenca-Ecuador

\begin{tabular}{|c|c|c|c|c|c|c|c|c|c|c|c|c|c|c|c|}
\hline & & I 2 & I 3 & I 4 & I 5 & I 6 & I 7 & I 8 & I 9 & I 10 & I 11 & I 12 & I 13 & I 14 & I 15 \\
\hline I 1 & $\mathrm{Rs}$ & .59 & .37 & .65 & .32 & .27 & .42 & .54 & .37 & .59 & .60 & .46 & .24 & .57 & .43 \\
\hline I 2 & $\mathrm{Rs}$ & & $\begin{array}{r}.43 \\
* * *\end{array}$ & $\begin{array}{l}.57 \\
* * *\end{array}$ & $\begin{array}{l}.34 \\
* * *\end{array}$ & $\begin{array}{l}.44 \\
* * *\end{array}$ & $\begin{array}{l}.48 \\
* * *\end{array}$ & $\begin{array}{l}.48 \\
* * *\end{array}$ & $\begin{array}{r}.34 \\
* * *\end{array}$ & $\begin{array}{l}.62 \\
* * *\end{array}$ & $\begin{array}{l}.71 \\
* * *\end{array}$ & $\begin{array}{r}.49 \\
* * *\end{array}$ & $\begin{array}{l}.32 \\
* * *\end{array}$ & $\begin{array}{l}.54 \\
* * *\end{array}$ & $\begin{array}{l}.33 \\
* * *\end{array}$ \\
\hline I 3 & $\mathrm{Rs}$ & & & $\begin{array}{r}.31 \\
* * *\end{array}$ & $\begin{array}{l}.51 \\
* * *\end{array}$ & $\begin{array}{l}.37 \\
* * *\end{array}$ & $\begin{array}{l}.41 \\
* * *\end{array}$ & $\begin{array}{l}.30 \\
* * *\end{array}$ & $\begin{array}{l}.51 \\
* * *\end{array}$ & $\begin{array}{l}.40 \\
* * *\end{array}$ & $\begin{array}{l}.43 \\
* * *\end{array}$ & $\begin{array}{c}.19 \\
*\end{array}$ & $\begin{array}{r}.34 \\
* * *\end{array}$ & $\begin{array}{l}.29 \\
* * *\end{array}$ & $\begin{array}{l}.21 \\
* * *\end{array}$ \\
\hline I 4 & $\mathrm{Rs}$ & & & & $\begin{array}{l}.37 \\
* * *\end{array}$ & $\begin{array}{l}.40 \\
* * *\end{array}$ & $\begin{array}{l}.37 \\
* * *\end{array}$ & $\begin{array}{l}.53 \\
* * *\end{array}$ & $\begin{array}{l}.35 \\
* * *\end{array}$ & $\begin{array}{l}.64 \\
* * *\end{array}$ & $\begin{array}{r}.74 \\
* * *\end{array}$ & $\begin{array}{l}.62 \\
* * *\end{array}$ & $\begin{array}{l}.25 \\
* * *\end{array}$ & $\begin{array}{l}.70 \\
* * *\end{array}$ & $\begin{array}{l}.43 \\
* * *\end{array}$ \\
\hline I 5 & $\mathrm{Rs}$ & & & & & $\begin{array}{r}.33 \\
* * *\end{array}$ & $\begin{array}{l}.43 \\
* * *\end{array}$ & $\begin{array}{r}.42 \\
* * *\end{array}$ & $\begin{array}{l}.62 \\
* * *\end{array}$ & $\begin{array}{r}.34 \\
* * *\end{array}$ & $\begin{array}{l}.44 \\
* * *\end{array}$ & $\begin{array}{l}.32 \\
* * *\end{array}$ & $\begin{array}{r}.57 \\
* * *\end{array}$ & $\begin{array}{r}.33 \\
* * *\end{array}$ & $\begin{array}{l}.30 \\
* * *\end{array}$ \\
\hline I 6 & $\mathrm{Rs}$ & & & & & & $\begin{array}{l}.29 \\
* * *\end{array}$ & $\begin{array}{r}.36 \\
* * *\end{array}$ & $\begin{array}{r}.30 \\
* * *\end{array}$ & $\begin{array}{l}.40 \\
* * *\end{array}$ & $\begin{array}{r}.39 \\
* * *\end{array}$ & $\begin{array}{l}.23 \\
* * *\end{array}$ & $\begin{array}{c}.20 \\
*\end{array}$ & $\begin{array}{r}.30 \\
* * *\end{array}$ & $\begin{array}{l}.22 \\
* * *\end{array}$ \\
\hline I 7 & $\mathrm{Rs}$ & & & & & & & $\begin{array}{l}.46 \\
* * *\end{array}$ & $\begin{array}{l}.48 \\
* * *\end{array}$ & $\begin{array}{l}.39 \\
* * *\end{array}$ & $\begin{array}{l}.48 \\
* * *\end{array}$ & $\begin{array}{l}.45 \\
* * *\end{array}$ & $\begin{array}{l}.44 \\
* * *\end{array}$ & $\begin{array}{r}.39 \\
* * *\end{array}$ & $\begin{array}{l}.26 \\
* * *\end{array}$ \\
\hline P 8 & Rs & & & & & & & & $\begin{array}{l}.47 \\
* * *\end{array}$ & $\begin{array}{l}.51 \\
* * *\end{array}$ & $\begin{array}{l}.57 \\
* * *\end{array}$ & $\begin{array}{r}.50 \\
* * *\end{array}$ & $\begin{array}{r}.39 \\
* * *\end{array}$ & $\begin{array}{l}.50 \\
* * *\end{array}$ & $\begin{array}{l}.42 \\
* * *\end{array}$ \\
\hline I 9 & Rs & & & & & & & & & $\begin{array}{l}.34 \\
* * *\end{array}$ & $\begin{array}{l}.42 \\
* * *\end{array}$ & $\begin{array}{l}.31 \\
* * *\end{array}$ & $\begin{array}{l}.71 \\
* * *\end{array}$ & $\begin{array}{l}.32 \\
* * *\end{array}$ & $\begin{array}{r}.36 \\
* * *\end{array}$ \\
\hline I 10 & Rs & & & & & & & & & & $\begin{array}{c}.75 \\
* * *\end{array}$ & $\begin{array}{l}.53 \\
* * *\end{array}$ & $\begin{array}{r}.33 \\
* * *\end{array}$ & $\begin{array}{l}.64 \\
* * *\end{array}$ & $\begin{array}{l}.34 \\
* * *\end{array}$ \\
\hline P 11 & Rs & & & & & & & & & & & $\begin{array}{l}.64 \\
* * *\end{array}$ & $\begin{array}{l}.35 \\
* * *\end{array}$ & $\begin{array}{l}.61 \\
* * *\end{array}$ & $\begin{array}{l}.47 \\
* * *\end{array}$ \\
\hline I 12 & Rs & & & & & & & & & & & & $\begin{array}{r}.39 \\
* * *\end{array}$ & $\begin{array}{l}.61 \\
* * *\end{array}$ & $\begin{array}{l}.42 \\
* * *\end{array}$ \\
\hline I 13 & Rs & & & & & & & & & & & & & $\begin{array}{r}.34 \\
* * *\end{array}$ & $\begin{array}{l}.36 \\
* * *\end{array}$ \\
\hline I 14 & Rs & & & & & & & & & & & & & & $\begin{array}{l}.35 \\
* * *\end{array}$ \\
\hline
\end{tabular}

$\mathrm{I}=$ ítem

$* p=.01 ; * * * p<.001$ 
Los descriptivos de cada ítem se detallan en la tabla 3, las puntuaciones oscilaron entre 1.95 y 2.93 con una media de 2.61 , todos los elementos tenían saturaciones aceptables (>.45), por lo que para el análisis factorial exploratorio se incluyeron los 15 ítems.

Tabla 3. Estadísticas de elemento Inventario Sistema de Creencias en Cuenca-Ecuador

\begin{tabular}{lcccc}
\hline Elemento & $\boldsymbol{M}$ & $\boldsymbol{D E}$ & $\begin{array}{c}\text { Correlación total de } \\
\text { elementos corregida }\end{array}$ & $\begin{array}{c}\boldsymbol{\alpha} \text { si el elemento } \\
\text { es suprimido }\end{array}$ \\
\hline 1 & 2.82 & .502 & .626 & .891 \\
2 & 2.73 & .639 & .666 & .888 \\
3 & 2.30 & 1.001 & .549 & .894 \\
4 & $\mathbf{2 . 9 3}$ & .346 & .676 & .892 \\
5 & 2.35 & .963 & .627 & .889 \\
6 & 2.48 & .960 & .454 & .898 \\
7 & 2.55 & .777 & .608 & .890 \\
8 & 2.77 & .633 & .656 & .888 \\
9 & 2.36 & .915 & .666 & .887 \\
10 & 2.81 & .535 & .671 & .889 \\
11 & 2.82 & .488 & .765 & .887 \\
12 & 2.78 & .573 & .596 & .891 \\
13 & $\mathbf{1 . 9 5}$ & 1.035 & .577 & .893 \\
14 & 2.86 & .445 & .627 & .892 \\
15 & 2.66 & .762 & .486 & .894 \\
\hline
\end{tabular}

$M=$ media; $D E=$ desviación estándar

En el análisis Factorial exploratorio mediante el método de extracción de componentes principales con una rotación ortogonal Varimax, se identificaron dos factores, que explicaron el $58.61 \%$ de la varianza. El primer factor: creencias y prácticas religiosas conformado, al igual que la versión original revisada (Holland et al., 1998) por los ítems: 4, 11, 10, 14, 1, 2, 12, 8, 15, 6 $(\alpha=.881)$ y una varianza explicada del $35.72 \%$. El segundo factor: apoyo social incluyó los ítems: $9,13,5,3$ y $7(\alpha=.883)$, con una varianza explicada del $22.89 \%$. Detalles en la tabla 4. (Insertar aquí Tabla 4) 
Tabla 4. Matriz de componente rotado del Inventario Creencias en Cuenca-Ecuador

\begin{tabular}{|c|c|c|c|c|}
\hline \multirow{2}{*}{ Elemento } & \multicolumn{2}{|c|}{ Factores } & \multirow{2}{*}{ Alpha } & \multirow{2}{*}{$\begin{array}{c}\text { \% varianza } \\
\text { explicada }\end{array}$} \\
\hline & 1 & 2 & & \\
\hline 4 & .849 & & \multirow{10}{*}{.881} & \multirow{10}{*}{35.72} \\
\hline 11 & .830 & & & \\
\hline 10 & .792 & & & \\
\hline 14 & .786 & & & \\
\hline 1 & .748 & & & \\
\hline 2 & .733 & & & \\
\hline 12 & .719 & & & \\
\hline 8 & .604 & & & \\
\hline 15 & .475 & & & \\
\hline 6 & .402 & & & \\
\hline 9 & & .858 & \multirow{5}{*}{.883} & \multirow{5}{*}{22.89} \\
\hline 13 & & .799 & & \\
\hline 5 & & .788 & & \\
\hline 3 & & .636 & & \\
\hline 7 & & .558 & & \\
\hline
\end{tabular}

\section{Validez concurrente}

La religiosidad de los participantes osciló entre 1 y 45 puntos con una media de 39.2 $(D E=7.1)$, por otra parte, la frecuencia de uso de estrategias de afrontamiento religiosas, uno de los dominios de la escala EAE-C osciló entre 1 y 12 puntos con una media de $8.98(D E=2.68)$. Se encontró una relación positiva fuerte $(r s=.569 ; p=.000)$ entre la religiosidad y la frecuencia de uso de estrategias de afrontamiento religiosas de la EAE-C, así se cumplió con la validez concurrente.

\section{Validez de constructo por el método de grupos conocidos}

En la tabla 5 se observa la existencia de diferencias significativas de la religiosidad y sus dominios según el sexo de los participantes $(p<.05)$, las mujeres son quienes mostraron mayor religiosidad que los hombres. Los resultados en estado civil y rangos etarios, según el tipo de adulto propuesto por Martín (2005), no fueron diferentes $(p>.05)$. 
Tabla 5. Religiosidad según características de los usuarios con enfermedades crónicas no transmisibles en Cuenca-Ecuador

\begin{tabular}{|c|c|c|c|c|c|c|c|}
\hline \multirow{2}{*}{$\begin{array}{l}\text { Característica } \\
M\end{array}$} & & \multicolumn{2}{|c|}{ Religiosidad } & \multicolumn{2}{|c|}{$\begin{array}{l}\text { Creencias y prácticas } \\
\text { religiosas y espirituales }\end{array}$} & \multicolumn{2}{|c|}{ Apoyo social } \\
\hline & & $D E$ & $M$ & $D E$ & $M$ & $D E$ & \\
\hline \multirow{3}{*}{ Sexo } & Hombres & 37.10 & 8.88 & 26.50 & 5.77 & 10.60 & 4.02 \\
\hline & Mujeres & 40.55 & 5.29 & 28.44 & 2.64 & 12.11 & 3.28 \\
\hline & $\mathrm{P}$ & \multicolumn{2}{|c|}{$.006^{*}$} & \multicolumn{2}{|c|}{$.027 *$} & \multicolumn{2}{|c|}{$.013^{*}$} \\
\hline \multirow{5}{*}{ Estado civil } & Soltero/a & 38.03 & 9.12 & 26.97 & 5.71 & 11.05 & 4.37 \\
\hline & Divorciado/a & 37.95 & 9.76 & 26.79 & 6.38 & 11.16 & 3.83 \\
\hline & Casado/a & 39.39 & 5.48 & 27.96 & 2.97 & 11.43 & 3.39 \\
\hline & Viudo/a & 41.20 & 5.81 & 28.60 & 2.80 & 12.60 & 3.31 \\
\hline & $\mathrm{P}$ & \multicolumn{2}{|c|}{.990} & \multicolumn{2}{|c|}{.864} & \multicolumn{2}{|c|}{.958} \\
\hline \multirow{5}{*}{ Tipo de adulto } & Jóvenes & 37.50 & 6.37 & 26.89 & 3.25 & 10.61 & 3.81 \\
\hline & Medios & 39.32 & 8.99 & 27.84 & 5.68 & 11.48 & 4.38 \\
\hline & Maduros & 39.81 & 6.19 & 27.85 & 3.37 & 11.95 & 3.35 \\
\hline & Mayores & 38.98 & 7.45 & 27.67 & 4.80 & 11.31 & 3.59 \\
\hline & $\mathrm{P}$ & \multicolumn{2}{|c|}{.419} & \multicolumn{2}{|c|}{.254} & \multicolumn{2}{|c|}{.446} \\
\hline
\end{tabular}

Jóvenes: de 20 a 39 años; Medios: de 40 a 49 años; Maduros: de 50 a 64 años: Mayores: > 65 años. $* p<.05$

\section{Discusión}

A nivel gramatical las validaciones psicométricas de los diferentes estudios siguieron un mismo patrón, al igual que las evaluaciones de carácter estadístico. Los valores de la consistencia interna global y de los factores I y II que reportan los estudios referenciados son más altos que los que se obtienen en esta investigación.

La validez convergente del análisis factorial exploratorio del ISC 15-R presentó correlaciones significativas (Holland et al., 1998), la validez concurrente para la versión italiana indicó una correlación moderada (Ripamonti et al., 2010). El instrumento ISC-C 15-R reportó una relación positiva fuerte entre la religiosidad y la frecuencia de uso de estrategias de afrontamiento religiosas de la EAE-C.

El alfa de Cronbach que reporta este estudio en el factor II es cercano al de la versión original e italiana (Holland et al., 1998; Ripamonti et al., 2010), en tanto que el de la adaptación realizada en Turquía es superior (Erci \& Aktürk, 2017).

En la mayoría de las adaptaciones psicométricas, al igual que en esta investigación se identificaron dos factores que no difirieron de la estructura de la versión original. La versión mexicana obtuvo tres factores (Almanza et al., 2000). La adaptación realizada en Alemania forzó la obtención de esta estructura (Grulke et al., 2003). 
En el estudio griego las mujeres informaron niveles significativos más altos de religiosidad que los hombres (Assimakopoulos et al., 2009), hallazgo que también obtiene este estudio. Este dato debe entenderse considerando factores biológicos, culturales y antropológicos.

En la adaptación colombiana el elemento seis se eliminó (Riveros et al., 2013) y el nivel de varianza explicada es cercano al de la versión ecuatoriana, en estudios realizados en otros países como en Estados Unidos (Holland et al., 1998), Alemania (Grulke et al., 2003) y Turquía (Erci \& Aktürk, 2017) la varianza explicada es más alta, demostrando una relación fuerte entre elementos del inventario.

En el elemento dos del ISC-C 15-R se adicionó una pregunta, que pretende conocer si la persona ora, medita o si práctica ambas técnicas. En este estudio los participantes utilizaban con mayor frecuencia la oración. Las opciones de respuestas de la versión ecuatoriana del inventario se adaptaron a los textos de los ítems traducidos al español. En este estudio, por medio de una ficha se recolectaron los datos sobre la tendencia religiosa, ninguno de los participantes respondió agnóstico.

\section{Conclusiones}

El inventario que evalúa SC religioso-espirituales, diseñado en Estados Unidos se validó psicométricamente en una población de personas con ECNT que asistían a contextos de salud en Cuenca-Ecuador, demostrando una muy buena consistencia interna.

Los elementos que conforman los factores del Inventario Sistemas de Creencias versión Cuenca Revisión 15 (ISC-C 15 R) se agruparon, modelando una estructura idéntica a la de la versión original. La validez concurrente y el método de grupos conocidos son procedimientos que respaldan la existencia del constructo teórico SC religiosas-espirituales en ecuatorianos. Las mujeres puntúan más alto que los hombres en todos los elementos del inventario, lo que quiere decir que son más religiosas y espirituales.

Los SC que asumen un monoteísmo se encuentran en el: zoroastrismo, judaísmo, cristianismo e islam. Esta perspectiva religiosa sobre el Theos ha sido introyectada por el mundo occidental moderno, en el caso del Ecuador, es asumida de manera consciente por un gran sector de personas que se denominan creyentes, en caso de que las personas signifiquen de manera distinta su visión del mundo, se encuentran influenciadas del mismo modo por los SC primarios.

Las variables: creencias y prácticas religiosas-espirituales y el apoyo social del grupo religioso-espiritual se encuentran presentes en todas las sociedades donde se evaluó este comportamiento.

El instrumento se ha puesto a prueba en varias culturas que utilizan distintos constructos lingüísticos que integran varios idiomas: hebreo, español, inglés, italiano, griego, turco y alemán.

El potencial espiritual, un sentimiento que acompaña al hombre milenariamente, motivó la fabricación de creencias sobre-naturales.

El hombre hábil, el ancestro más antiguo del hombre sabio, desarrolló una psicología basada en símbolos una vez que su organismo se encontraba tanto morfo-anatómica como cerebralmente apto para ello. El símbolo sería el producto sofisticado y complejo del contacto establecido entre el hombre arcaico y su medio ambiente.

Los primeros sistemas simbólicos evolucionaron hacia sistemas de creencias, los símbolos son las estructuras más simples de los SC y especialmente de los religiosos. Los SC espiritua- 
les-religiosos son construcciones cognitivas moldeados por la naturaleza, el tiempo y la cultura en el cual evolucionan los grupos biológicos pertenecientes a ramas homínidas.

Para esta investigación, se consideraron tres enfermedades crónicas por la necesidad de contar con instrumentos psicométricos validados que midan rasgos religiosos y espirituales en diversos usuarios que asisten a hospitales y centros de salud. Las experiencias oncológicas por definición son más complejas que las enfermedades diabetes o hipertensión.

La investigación en psicología de enlace en el Ecuador tiene que crear herramientas de evaluación basadas en investigación científica, crítica, ética, y sobre todo, objetiva, para que estos instrumentos se apliquen en procesos psicoterapéuticos de carácter clínico psicológico y médico. Además, puede y debe, encontrar una aplicación en el ámbito educativo.

\section{Referencias bibliográficas}

Almanza, J., Monroy, M., Bimbela,A., Payne, D., \& Holland, J. (2000). Academy of Psychosomatic Medicine: Proceedings of the 46th Annual Meeting November 18-21, 1999, New Orleans, LA. En Spanish Version of the Systems of Belief Inventory (SBI-15-R): Cross Cultural Research on Spiritual and Religious Beliefs (158). Psychosomatics, 41(2), 158. http://doi. org/10.1176/appi.psy.41.2.157

Arnold, M., \& Osorio, F. (1998). Introducción a los conceptos básicos de la teoría general de los sistemas. Cinta de Moebio. Revista de epistemología de las ciencias sociales, (3), 40-49. Disponible en: https://www.redalyc.org/pdf/101/10100306.pdf

Assimakopoulos, K., Karaivazoglou, K., Ifanti, A., Gerolymos, M., Kalofonos, H., \& Iconomou, G. (2009). Religiosity and its relation to quality of life in Christian Orthodox cancer patients undergoing chemotherapy. Psycho-Oncology, 18(3), 284-289. http://doi.org/10.1002/ pon. 1402

Baider, L., Holland, J., Russak, S., \& De-nour, A. (2001). The system of belief inventory (SBI15): A validation study in Israel. Psycho-Oncology, 10 (6), 534-540. http://doi.org/10.1002/ pon.554

Bernal, L. (2017). Aproximación neurocientífica a la esfera religiosa. Estrategias de afrontamiento espiritual y calidad de vida en personas que presentan experiencias oncológicas. Maskana, 0, 50-76. Disponible en: https://publicaciones.ucuenca.edu.ec/ojs/index.php/maskana/ article/view/1877

Bernal, L. (2018). Adaptación y Validación Psicométrica de la Escala Estrategias de Afrontamiento Espiritual en Cuenca-Ecuador. Revista Médica HJCA, 10(3), 228-234. Disponible en: https://doi.org/dx.doi.org/10.14410/2018.10.3.ao.37

Boyer, P. (2001). Religion Explained: The Evolutionary Origins of Religious Thought. New York, United States of America: Basic Books

Costello, A., \& Osborne, J. (2005). Best practices in exploratory factor analysis: four recommendations for getting the most from your analysis. Practical Assessment, Research \& Education, 10(7), 1-9

Eliade, M. (1999). La búsqueda: historia y sentido de las religiones. En Colodrón, A. (trad.). Barcelona, España: Kairós 
Erci, B., \& Aktürk, Ü. (2017). The System of Belief Inventory: A Validation Study in Turkish Cancer Patients. Journal of Religion and Health, 57(4), 1237-1245. doi: http://doi. org/10.1007/s10943-017-0406-0

Feixas, G., \& Miró, M. (1993). Aproximaciones a la psicoterapia. Una introducción a los tratamientos psicológicos. Barcelona, España: Paidós Ibérica

Friedberg, R., \& McClure, J. (2005). Práctica clínica de terapia cognitiva con niños y adolescentes. Barcelona, España: Paidós Ibérica

Gorsuch, R. (1983). Factor analysis ( $2^{\mathrm{a}}$ Ed.). Hillsdale, United States of America: Lawrence Erlbaum Associates

Grulke, N., Bailer, H., Blaser, G., Geyer, M., Brahler, E., \& Albani, C. (2003). Measuring religious attitudes: reliability and validity of the German version of the Systems of Belief Inventory (SBI-15R-D) in a representative sample. Mental Health, Religion \& Culture, 6(3), 203-214. doi.org/10.1080/1367467031000087665

Hair, R., Tatham, R., Anderson, R., \& Black, W. (1998). Multivariate data analysis- Upper Saddle River, New Jersey, United States of America: Prentice-Hall

Hambleton, R. K. (1996). Adaptación de tests para su uso en diferentes idiomas y culturas: fuentes de error, posibles soluciones y directrices prácticas. En J. Muñiz (Coor.), Psicometría. Madrid: Universitas

Hampel, N., Brähler, E., Zenger, M., Albani, C., \& Heuft, G. (2019). Psychometrische Überprüfung der Kurzversion des ,Systems of Belief Inventory“ in einer deutschlandweiten repräsentativen Bevölkerungsstichprobe. PPmP-Psychotherapie, Psychosomatik, Medizinische Psychologie. https://doi.org/10.1055/a-0865-2211

Holland, J., Kash, K., Passik, S., Gronert, M., Sison, A., Lederberg, M., ... Fox, B. (1998). A Brief spiritual beliefs inventory for use un quality of life research in file-threatening illness. Psycho-Oncology, 7, 460-469. http://dx.doi.org/10.1002/(SICI)10991611(199811/12)7:6<460::AID-PON328>3.0.CO;2-R

Martín, J. (2005). Los factores definitorios de los grandes grupos de edad de la población: tipos, subgrupos y umbrales. Scripta Nova: Revista Electrónica de Geografía y Ciencias Sociales, 9 (2). Recuperado dehttps://www.raco.cat/index.php/ScriptaNova/article/view/55937/65243

Morris, B. (1995). Introducción al estudio antropológico de la religión. Barcelona, España: Ed. Paidós

Ries, J. (2016). Le origini delle religioni. E. Nussio (trad.). El origen de las religiones. Madrid, España; Libsa

Ripamonti, C., Borreani, C., Maruelli, A., Proserpio, T., Pessi, M., \& Miccinesi, G. (2010). System of belief inventory (SBI-15R): a validation study in Italian cancer patients on oncological, rehabilitation, psychological and supportive care settings. Tumori, 96, 1016-1021

Rivera, A. (1998). Arqueología del lenguaje en el proceso evolutivo del género homo. Espacio, tiempo y forma, Serie I, Prehistoria y Arqueología, (11), 13-43. Disponible en: http:// revistas.uned.es/index.php/ETFI/article/view/4664/4503

Riveros, F., Bernal, L., Bohórquez, D., Vinaccia, S., \& Quiceno, J. (2013). Inventario de sistema de creencias (SBI-15R) en Colombia: estructura factorial y confiabilidad en población universitaria y pacientes crónicos. Revista Colombiana de Enfermería, 17, 13-20. https:// doi.org/10.18270/rce.v17i13.2103

(C) Psy, Soc, \& Educ, 2020, Vol. 12(2) 
Saiz, M., \& Amézaga, P. (2005). Psiconeurociencia y arquetipos-construyendo un diálogo entre psicología analítica y neurociencia. Psicologia USP,16(3), 95-117

Sztajer, S. (2013). The concept of homo religiosus and its philosophical interpretations. Anglojęzyczny Suplement Przegladu Religioznawczego, 1, 17-27. Recuperado de http:// www.ptr.edu.pl/images/Suplement_ang/2013 no. 1/2. Sławomir Sztajer.pdf

Usó-Doménech, J., \& Nescolarde-Selva, J. (2016). What are Belief Systems ? Foundations of Science, 21, 147-152. https://doi.org/10.1007/s10699-015-9409-z

Yalom, I. (1998). The Yalom reader. En J. Bayo (trad.). Psicoterapia existencial y terapia de grupo (12 ${ }^{\text {va }}$ Ed.). Barcelona, España: Paidós Ibérica 